January 16, 2003

\title{
Quasiperiodic pulsations in a solar microwave burst
}

\author{
V. V. Grechnev ${ }^{1,2}$, S. M. White ${ }^{2}$ and M. R. Kundu ${ }^{2}$ \\ ${ }^{1}$ Institute of Solar-Terrestrial Physics, Lermontov St. 126, Irkutsk, Russia 664033 \\ ${ }^{2}$ Astronomy Department, University of Maryland, College Park, MD 20742 \\ grechnev, kundu, white@astro. umd.edu
}

\begin{abstract}
Quasiperiodic pulsations in solar flares can provide important information on physical conditions in flaring regions. In this paper we study a microwave burst that showed deep quasiperiodic pulsations. The most dramatic feature of this event has been discussed by Asai et al. (2001): in the second of four bursts during the flare strongly modulated pulsations appear in radio images from the eastern end of a long loop and in hard X-rays from the western end of the loop. In this paper we show in addition that (i) at least five distinct radio sources with very different time profiles can be identified, including emission from the long loop connecting the modulated radio and X-ray sources; (ii) substructure is also present in the radio emission from the eastern end of the long loop during the first burst of the flare, but with timescales shorter than in the second burst; (iii) radio modulations are seen at the western end of the loop during the second burst, but at a level some 20 times weaker than at the eastern end; (iv) these radio modulations at the western end of the loop, like the hard X-ray modulations at the same location, appear to lead the modulations at the eastern end by about 0.5 $\mathrm{s}$, but all have the same period. The period of the modulation can be explained by MHD oscillations of the loop $\approx 120^{\prime \prime}$ long connecting the sources: both oscillations that change the magnetic field strength in the loop, such as propagating fast mode waves, and torsional oscillations that change the direction of the magnetic field in the loop can explain the observed properties of the modulation of the radio emission. An impulsive reconnection episode is a plausible source of oscillating fast-mode waves and is consistent with some other aspects of the event. However, it is difficult to reconcile the strength of the radio modulations at the eastern end of the loop with their delay relative to the emissions at the western end where the modulation is observed to be much weaker: if the electrons originate at the
\end{abstract}


western end where the main energy release seems to occur, and the modulation is imposed on them there before they propagate $10^{10} \mathrm{~cm}$ to the other end of the loop, any effects due to a spread in electron energies or pitch angles would lead to a spread in propagation times that should smooth out the modulation of the radio emission from the remote source, as should any trapping of electrons in the loop, yet the radio emission from the eastern end of the loop shows much stronger modulation than the radio and hard X-ray emission from the western end of the loop.

Subject headings: Sun: flares - Sun: corona - Sun: radio radiation

\section{INTRODUCTION}

Solar oscillatory phenomena have played an important role in placing constraints on physical parameters in the solar atmosphere. Quasi-periodic pulsations (QPP) in coronal loops as observed in radio emission as well as in other spectral domains were reviewed by Aschwanden (1987) and Aschwanden et al. (1999). Of special interest are the QPP observed in solar flares, because they may give some additional insight into flare conditions. Asai et al. (2001) presented an unusual example of QPP observed in microwaves at $17 \mathrm{GHz}$ by the Nobeyama Radioheliograph $(\mathrm{NoRH})$ with a modulation depth of $>70 \%$. These QPP's have a counterpart, less strongly modulated, in hard X-ray (HXR) emission observed by the Hard X-ray Telescope (HXT) on the Yohkoh satellite. The authors propose that this event is an example of periodic acceleration of electrons due to a Fermi process between two shocks,

one being fixed, and the other being associated with an oscillating magnetic loop. Here we investigate the detailed properties of this event further.

Quasi-periodic pulsations have been observed many times in the past both in radio and in hard X-ray emissions (see, e.g., Rosenberg 1970; Altyntsev et al. 1998; Altyntsev et al. 1999; Kundu et al. 2001), but we are aware of few other microwave events with such a high degree of modulation depth in the pulsations observed. Besides the event of November 10, 1998 which we are discussing in this paper, we can refer to the events reported by Tapping (1983) and Nakajima et al. (1983).

There are several questions to be answered with regard to this pulsation event, namely the cause of the prominent radio pulsations, their relation to the X-ray pulsations and why this event is exceptional in nature. We ask if these extraordinary pulsations reflect any unusual phenomenon associated with special conditions in the flare region. This event as observed in the radio domain consists of a sequence of several bursts with a total duration 
of order 5 minutes. We find that the first impulsive burst, which occurred 2 minutes before the burst with strongest QPP, also showed similar substructure. However, the timescales of these pulsations were shorter than in the main burst. We also find that oscillations are seen in the radio emission from the far end of a loop connecting to the main source of QPP radio oscillations, but at a level some 20 times weaker. The relative timing of the oscillations at the two ends of this long loop is used to discuss the possible origins of the oscillations.

\section{THE OBSERVATIONS}

The flare was of GOES importance 1N/C7.9 and occurred in active region AR8375, close to the western limb, on 1998 November 10 at 00:12 UT. It was observed by NoRH, the radio polarimeters at Nobeyama (NoRP), the Extreme-ultraviolet Imaging Telescope (EIT) on the SOHO satellite, the Soft X-ray Telescope (SXT) and HXT on Yohkoh, but not by the Compton Gamma Ray Observatory detectors or by the Transition Region and Coronal Explorer (TRACE). Magnetogram data are available from Michelson Doppler Imager (MDI) on $\mathrm{SOHO}$ at $03 \mathrm{UT}$. We used high-cadence $(0.1 \mathrm{~s}) \mathrm{NoRH}$ image sets which are available during the first burst. The quality of $34 \mathrm{GHz}$ images is often poor for this event, and the microwave sources are faint at $34 \mathrm{GHz}$. There is no evidence for an eruption in this event either in EIT or the SOHO Large Angle Spectroscopic Coronagraph (LASCO) data which cover the time of the flare. Hiraiso Observatory radio spectra also do not show any detectable activity in the frequency range 25 to $2000 \mathrm{MHz}$ during this event. No detectable response is present in Hiraiso single-frequency records at 500 and $2800 \mathrm{MHz}$. From these facts we conclude that the flare was not associated with any ejection. SXT and EIT images show AR8375 to be a complex multi-loop system with sequential brightening of loop-like structures. Three small events occurred in this region in the 2 hours prior to the event of interest in this paper. Asai et al. (2001) analyzed and interpreted the most prominent pulsating part of the event and estimated plasma parameters. Here we analyze substructure in an earlier part of the event as well as additional components.

\subsection{Microwave time profiles}

Time profiles of the entire event recorded by NoRH at $17 \mathrm{GHz}$ and by Yohkoh/HXT in all four bands are shown in Figure 1. The event was complex and consisted of several consecutive enhancements (bursts) observed in X-rays and microwaves. The first burst occurred at 00:12:09-00:13:00 with a peak flux of $220 \mathrm{sfu}$ at $17 \mathrm{GHz}$. The radio light curves show fine structure during this burst superimposed on a classic impulsive-phase spike. The second 
burst with deep pulsations occurred at 00:14:17-00:15:04 with a peak $17 \mathrm{GHz}$ flux of $26 \mathrm{sfu}$. The third burst occurred at 00:15:04-00:16:05 and is distinct in the radio light curves but not in hard X-rays. The fourth burst, at 00:16:09-00:16:37, was very weak at radio wavelengths.

Nobeyama polarimeter data at fixed frequencies of 2, 3.75, 9.4, 17 and $35 \mathrm{GHz}$ show that the initial impulsive spike has a peak frequency between 9 and $17 \mathrm{GHz}$. The high-frequency (optically thin) microwave spectral index from 17 to $35 \mathrm{GHz}$ is -3.5 . Since the polarimeter data are less sensitive than the radioheliograph, the data for the other peaks are inconclusive but they appear to have spectra similar to the main peak. The modulations in the second peak are clearly visible at $9.4 \mathrm{GHz}$.

Asai et al. (2001) estimated the microwave spectral index for the second burst in the optically thin part of the spectrum to be -1.5 to -2 from the polarimeter data, which have, however, poor signal/noise ratio at $35 \mathrm{GHz}$. An alternative estimate can be made using NoRH data at 17 and $34 \mathrm{GHz}$, which indicate that the microwave spectral index in the second event is steeper and similar to that of the first spike, at about -3 .

\subsection{Hard X-ray time profiles}

The initial impulsive spike is detected in all 4 HXT channels (Fig. 1) and has a hard $\mathrm{X}$-ray spectrum that is harder than the subsequent peaks. The second and third peaks show considerable structure in the hard X-ray time profiles, with the modulations in the second peak clearly visible in the HXT M1 channel (23-33 keV) but showing less modulation than the radio. There is considerable similarity between the $17 \mathrm{GHz}$ microwave emission and the Yohkoh/M2 HXR emission.

The first peak (01:12:10-01:12:40) is dominated by a short spike of $3.5 \mathrm{~s}$ (FWHM) prominent in the $\mathrm{H}$ and M2 channels, which peaked at 00:12:27. This spike is superposed on top of a longer lasting emission of about $25 \mathrm{~s}$ for the M2 and $\mathrm{H}$ channels. The power-law photon spectral index $\gamma$ during the first burst is about 3.6, corresponding to an electron spectral index of $\delta=\gamma+1.5=5.1$ from the $\mathrm{H} / \mathrm{M} 2$ ratio. The other channel ratios give $\gamma=$ 3.3 from M2/M1 and $\gamma=3.1$ from M1/L. A longer decay is present in M1 and especially in the L channel up to about 00:13:10. This "shoulder" has a somewhat softer hard X-ray spectrum $(\gamma=4.1)$. The spectrum and the time profile of the first peak suggest an almost entirely nonthermal origin for the emission. The subsequent peaks have much softer spectra, with the third burst almost matching the first peak in the $\mathrm{L}$ channel. The fourth burst (01:16:10-01:16:40) is much more prominent in hard X-rays than in microwaves and appears to have a harder spectrum than the second and third bursts. 


\subsection{Soft and hard X-ray imaging data}

X-ray images of the event are shown in Figure 2. The preflare image (panel a) shows two compact loops to the north-east and south-west of the region, together with a long high loop (labelled $\mathrm{L}_{C}$ ) connecting the north-east to the center of the active region. In Fig. $2 \mathrm{~b}$ we approximately reproduce the Yohkoh/SXT Be119 flare image at 00:14:32 (during the second burst) as shown by Asai et al. (2001). This image is dominated by the small loop to the south-west of the region. The spatial configuration observed in hard X-rays is simple. Hard X-ray images show a single compact feature during the inital impulsive spike at all channels. Contours in panel b indicate the location of the HXT/M2 source, which appears to be at the south end of the soft X-ray loop.

In Fig. 2c we present an image of the flare region composed of several SXT frames obtained well after the event, from 00:19:48 through 00:21:34 UT. Saturated parts of several long-exposure frames were substituted with nonsaturated pixels from frames of shorter exposure time. Further, the frames were averaged and interpolated. This image shows a loop $\mathrm{L}_{A}$ connecting the southern end of the compact north-east preflare loop with the main SXT loop $\mathrm{L}_{B}$ to the south-west. This loop is also visible in Fig. $2 \mathrm{~b}$.

The images in Fig. 2a-c show several long and short loops. A sketch of the configuration is shown in Fig. 2d. The brightest loop $\mathrm{L}_{B}$ is a compact loop observed in the southwestern region (denoted as source "B" in the paper by Asai et al. 2001), with the hard X-ray source being displaced southward from the brightest soft X-ray region to coincide with the $\mathrm{SW}$ footpoint of $\mathrm{L}_{B}$. The orientation of the lower part of the loop $\mathrm{L}_{A}$ 's northeastern leg is magnified, in order to emphasize the shape of the entire loop. Note that the top of loop $\mathrm{L}_{A}$ may be oriented along the line of sight; this will be important in interpreting the radio images.

From SXT data we produced temperature and emission measure maps. The density distribution in the long loop $\mathrm{L}_{A}$ is rather uniform, whereas it is non-uniform in the short loop $\mathrm{L}_{B}$. The main SXR emitting region is a bright kernel of dimension $7 \times 10^{8} \mathrm{~cm}$, and its area is $6 \times 10^{17} \mathrm{~cm}^{2}$. This bright kernel occupies about $25 \%$ of the entire area of the short loop. Assuming a depth of the same dimension, i.e. $8 \times 10^{8} \mathrm{~cm}$, we obtain a volume of $5 \times$ $10^{26} \mathrm{~cm}^{3}$. We used these values to obtain estimates for the plasma density from GOES data when the SXT frames were saturated. The results will be used in section 4 . 


\subsection{Microwave images}

Figure 3 shows $17 \mathrm{GHz}$ radio images of the flare at four representative times made from NoRH data. These images are $1 \mathrm{~s}$ snapshots deconvolved in the AIPS package and clean components are restored with a circular $10^{\prime \prime}$ beam. Image-to-image motion has been removed by coalignment of appropriate features, and the resulting sequence of images is believed to be aligned to within better than the $3^{\prime \prime}$ pixel size. The four times shown are (a) during the initial impulsive peak, (b) during the period between the first and second bursts, (c) during the second burst at the peak of an oscillation, and (d) during the fourth burst. The contours show images that have had the pre-burst emission subtracted, with the pre-burst image shown as the underlying greyscale in each case. These images show a number of labelled sources, also indicated in Fig. 3. Five sources are shown in the top left panel, which shows contours at 00:12:27, near the peak of the first impulsive burst. Prior to the flare the brightest radio source is in the north-east of the region, labelled " 1 ". The flare sources include (i) emission extended from source 1 to the south-west (panel a), where source "2", which is the source showing the oscillations (panel c), is located; (ii) a source extended east-west, labelled "3" (panel b), whose eastern end is close to the oscillating source; (iii) another extended source, labelled "4", that is coincident with the compact bright soft X-ray loop visible in the SXT images in the south-west of the region (Fig. 2); and (iv) a compact source, "5", that lies at the southern end of the loop containing source 4 and is coincident with the hard X-ray source seen in the HXT images. Source 5 is the location of the radio emission during the fourth burst (panel d). Sources 4 and 5 are very close to one another and cannot always be separated in the analysis of the event.

In order to identify the radio sources with optical and X-ray features, Figure 4 shows overlays of the radio emission on optical continuum (from SOHO/MDI) and soft X-ray (from Yohkoh/SXT) images. Sources $1 \& 2$ lie near the negatively-polarized sunspot to the northeast of the region. Source 3 is identified with the long soft X-ray flare loop $\mathrm{L}_{A}$ that connects sources 2 (the main radio source) and 4/5 (the main soft and hard X-ray source). Figure 4 indicates that the entire loop $\mathrm{L}_{A}$ is visible as a radio source, but the apparent top of the loop where it bends over is much weaker than the two sides of the loop in the radio images, whereas in the X-ray images the loop top and the legs are of similar brightness. We interpret this result as a combination of the viewing-angle effect and the possibly weaker magnetic field at the top of the loop compared to the footpoints (Dulk 1985): at the top of the loop we are looking nearly along the magnetic field, and the magnetic field strength there is likely to be lower than at the footpoints. Both these effects act to diminish the gyrosynchrotron emissivity at the loop top compared to the legs where we are looking more orthogonal to the magnetic field direction. 
Figure 5 shows $17 \mathrm{GHz}$ radio light curves for sources $2-5$ at $1 \mathrm{~s}$ time resolution (the light curve for source 1 is essentially identical in morphology to that of source 2). In addition, 34 $\mathrm{GHz}$ light curves are shown for the two brightest sources, 2 and 4 . The $34 \mathrm{GHz}$ emission is relatively weak in this event and although sources 3 and 5 are detected at $34 \mathrm{GHz}$, they are too weak for reliable light curves to be derived. The pixels used to determine the light curves for sources 4 and 5 in this figure are chosen to emphasize their differences, i.e., for source 4 they are pixels that are only radio-bright during the first burst, while for source 5 they are pixels that are bright during the fourth burst. Fig. 5 shows a number of remarkable features:

1. In addition to the modulation studied by Asai et al. (2001), source 2 shows 4 evenly spaced peaks during the initial impulsive burst.

2. Source 4 brightens in the initial burst at the same time as source 2 , but decays much more rapidly. Its time profile in the initial burst is a smooth rise and fall; source 4 is not bright in the radio at later times.

3. Source 5, which is adjacent to source 4 and appears to be at the southern footpoint of a soft X-ray loop, has a sharp rise and fall like that of source 4 during the initial burst, but also shows emission during the subsequent bursts, most noticeably during the fourth burst at 00:16:20 when none of the other radio sources show emission.

4. Source 3 shows emission with a time profile similar to that of source 2 during the initial burst, but decays much more slowly so that it is prominent during the period between the first and second bursts; it shows no sign of the oscillations.

5. At $34 \mathrm{GHz}$ emission is only seen during the initial impulsive burst, when source 2 does show the discrete peaks seen at $17 \mathrm{GHz}$.

6. At $34 \mathrm{GHz}$, source 4 is brighter than source 2 in the initial burst, whereas at $17 \mathrm{GHz}$ source 2 is brighter.

Note that Asai et al. (2001) discussed sources 1, 2 \& 3 together as their "source A", and discussed our sources $4 \& 5$ as their source "B", but the differences in the lightcurves of sources $2-5$ indicate that they need to be identified as separate sources.

\subsection{Radio polarization}

All the sources are left circularly polarized. Figure $2 \mathrm{~d}$ indicates the polarity of the underlying photospheric magnetic field, which shows that sources $1,2 \& 3$ lie over regions 
of downgoing magnetic field and sources $4 \& 5$ lie over upgoing magnetic field. We therefore expect sources 1, $2 \& 3$ to be left circularly polarized, but not sources $4 \& 5$ : since the emission from these sources must pass through the neutral line above the active region on its path to us, we assume that their polarization is reversed there due to mode coupling (e.g., White et al. 1992).

The emission from source 1 is about $30 \%$ polarized both before and during the first impulsive burst, then $40 \%$ polarized during the second and third bursts; the flare emission from sources 2 and 3 is $35 \%$ polarized during the first impulsive burst and $\sim 45 \%$ during the second and third bursts; source 4 is $25 \%$ polarized at the flare peak and its degree of polarization is strongly anticorrelated with flux during the initial spike; and source 5 is around $40 \%$ polarized during the first and third bursts but $25 \%$ during the fourth burst.

During the strong modulations in the second burst, source 2 shows a degree of polarization constant to within a few percent. As with source 4, source 2 shows some anticorrelation between flux and degree of polarization during the first burst. This anticorrelation could be an optical depth effect: a gyrosynchrotron source should become less polarized when its optical depth exceeds unity, because the $x$ mode is already optically thick and its flux does not change with an increase in the number of nonthermal electrons, whereas the $o$ mode may still be optically thin and will increase in flux, thereby decreasing the overall degree of polarization.

\subsection{Spectral information}

The turnover frequency of the overall microwave spectrum was at $12-13 \mathrm{GHz}$, indicating that the magnetic field in the source was rather strong. We can infer spectral information for sources 2 and 4 from Fig. 5. The radio flux spectral index from 17 to $34 \mathrm{GHz}$ for source 2 is -3.0 , corresponding to an electron energy spectral index of -4.7 , while for source 4 the radio spectral index is $\sim-1.8$ (electron energy spectral index of -3.4 ). We argue that all of the radio sources discussed here are nonthermal and thus represent electrons accelerated to nonthermal energies by the flare. For source 3, which is sufficiently weak that we cannot easily measure its radio spectrum, we note that as a radio source it is similar in strength to source 4 once the initial impulsive spike ends, but in soft X-rays source 4 is far brighter than source 3, so source 3's radio emission cannot be due to thermal bremsstrahlung which would require source 4 to be a much brighter radio source than source 3. Asai et al. (2001) argued that the flux spectrum of their source B, equivalent to our sources $4 \& 5$, during the second burst was flat and therefore consistent with optically thin thermal bremsstrahlung from the brightest soft X-ray loop visible during the second burst. Further, the flux, of order 1-2 sfu, 
is consistent with the thermal bremsstrahlung radio flux expected from the measured soft X-ray emission measure and temperature (1.2 sfu based on GOES data). We find that the $17 \mathrm{GHz}$ flux from source 4 during the second burst is slightly larger (of order $50 \%$ ) than the $34 \mathrm{GHz}$ flux. Some of this flux must be due to the thermal bremsstrahlung from the soft $\mathrm{X}$-ray emitting gas, but this cannot be true of the modulated component that arises just to the west of the soft X-ray loop, as shown in Fig. 3c, since its time profile is very different from that of the predicted thermal bremsstrahlung component.

Note that the electron spectral index inferred from the radio emission from source 2 is very similar to that inferred from the hard X-rays $(\sim-4.8)$ : however, the spatial location of the hard X-ray emission is not source 2 but is closer to sources $4 \& 5$, whose radio electron spectral index $(-3.4)$ is much flatter than that of the HXR-emitting electrons.

\section{RADIO AND X-RAY MODULATION}

In Figure 6 we show detailed expanded $17 \mathrm{GHz}$ radio and 23-33 keV hard X-ray time profiles during the first and second impulsive bursts. The curves are all normalized to a peak of unity. During the first burst flare-mode data are available with $0.1 \mathrm{~s}$ time resolution, and these are used in the upper plot. During the second burst only data with $1 \mathrm{~s}$ time resolution are available. The coalignment procedures for consecutive frames are accurate to better than one pixel $\left(3^{\prime \prime}\right)$ for the $1 \mathrm{~s}$ cadence images $\left(10^{\prime \prime}\right.$ beam) mapped using the AIPS software. The restoring beam size is larger for the $0.1 \mathrm{~s}$ images, so we do not present separate lightcurves for all five emitting regions, but focus on the two brightest regions, sources 2 and $4 / 5$.

The bottom panel of Fig. 6 shows the second burst with the prominent modulations located at source 2 already discussed by Asai et al. (2001). We confirm their findings, but in addition the analysis of spatially resolved light curves shows that there is a small component of radio emission near the hard X-ray source that does show modulated radio emission, as seen in the light curve for "source 4" plotted in the figure. This emission is at a level some 20 times weaker than the modulated radio emission from source 2, but is clearly present: three of the peaks match those from source 2. However, the spatial location of this emission is not the same as that of source 4 during the first burst, but rather a source just to the west that is clearly elongated north-south, with the southern edge being roughly coincident with

source 5 (Fig. 3c). We have looked for differences in the light curves of the upper and lower halves of this elongated source but the data do not show any differences that are significant to within the noise. We will refer to this emission as source 4 for the rest of this section.

Asai et al. (2001) analyzed the light curves for the second burst and inferred an $\sim 0.5 \mathrm{~s}$ 
delay of the $17 \mathrm{GHz}$ emission from source 2 relative to the hard X-ray emission from source 4. Asai et al. (2001) argue that this delay implies that the nonthermal electrons responsible for the $17 \mathrm{GHz}$ emission from source 2 originated at source 4 and propagated along loop $\mathrm{L}_{A}$ to the remote site: this loop is at least $120^{\prime \prime}=10^{10} \mathrm{~cm}$ long, not including projection effects, and $0.5 \mathrm{~s}$ is a plausible propagation time for relativistic nonthermal electrons (albeit not for the $\sim 100 \mathrm{keV}$ electrons that produce the HXT M1 hard X-ray emission). In this scenario, we expect that the radio emission from close to the hard X-ray source should not show a significant delay relative to the hard X-rays, and the data in Fig. $6 \mathrm{~b}$ are consistent with this idea: the $17 \mathrm{GHz}$ light curve for source 4 shows no delay relative to the HXT M1 light curve. However, it is also apparent that the inferred delay for the radio emission from source 2 is entirely attributable to the two brightest radio peaks (the second and third of the four most prominent peaks); in the first prominent peak both radio light curves peak at the same time as the X-rays, while in the fourth peak the radio emission from source 2 leads both the radio emission from source 4 and the hard X-ray emission. In summary, we do find modulations in the radio emission from the vicinity of the hard X-ray source that match the hard X-ray modulation and may occur about 0.5 s earlier than the deep modulations in source 2 .

In the initial impulsive burst, shown in the top panel of Fig. 6, the M1 hard X-rays and the radio emission from both sources 2 and 4 peak at the same time, but otherwise show major differences in their behaviour. Source 4 decays almost immediately after the peak, while source 2 remains high and HXT M1 decays only gradually. The $1 \mathrm{~s}$ cadence data for source 2 (Fig. 5) seem to show quasiperiodic oscillations with a period of order $5 \mathrm{~s}$ that is shorter than the period of the modulations in the second burst. At the 0.1 s cadence of Fig. 6 , it can be seen that the structure of the light curve for source 2 is more complex. During the rise to maximum there is structure at $2 \mathrm{~s}$ scales (four main peaks, three consecutive peaks with $1.6 \mathrm{~s}$ separation), while after the maximum there are three individually narrow peaks (including the highest peak) with separations of $4.4 \mathrm{~s}$. It is possible that these timescales represent oscillation periods and that the period increases from the rise to the decline phase, but we regard the data as insufficient (too few cycles - in each case the removal of one peak destroys the periodicity) to make this case. If these are modulations as in the second burst, the modulation depth is much smaller in the first burst.

It is notable that there is fine structure in the radio emission from source 4 during the rise of the first burst but not during the decay. Some of this fine structure matches similar structure in the light curve of source 2 with a delay of order $0.4 \mathrm{~s}$, but most of the structure in the rise of the two light curves does not match. There is no sign of fine structure in the abrupt decay of source 4. Since Asai et al. (2001) find a delay of $0.5 \mathrm{sec}$ of the radio emission from source 2 with respect to the hard X-rays from source 4 in the second burst, we have inspected the higher time resolution radio fluxes for features common to the radio and X-ray 
light curves during the first burst but do not find sufficient common features to measure any delays present.

\section{DISCUSSION}

Two bundles of magnetic field lines ("loops") are prominent in this flare: the compact loop $\mathrm{L}_{B}$ that dominates both the soft X-rays and the hard X-rays, with the hard X-rays apparently coming from near the southern footpoint; and the long loop $\mathrm{L}_{A}$, with the main radio source at its northern footpoint. The phenomena in this event that require understanding include the following:

1. The coherent, highly modulated radio oscillations at source 2 in the second burst, addressed by Asai et al. (2001).

2. The fact that hard X-ray oscillations are also seen, but at the opposite end of a long loop from the radio emission. Modulated radio emission does occur in this location, but at a level 20 times weaker than at the other end of the loop. The loop itself is visible both in soft X-rays and in radio.

3. The nature and interpretation of delays between the radio and hard X-rays.

4. The apparent occurrence of fine structure (in the radio light curves only), with a shorter period, at the same location (source 2) during the first burst.

5. The fact that the radio emission from the main soft and hard X-ray source (source 4) during the first burst terminates before the emission from source 2 .

6. What is the connection between the two loops?

The modulations can be intrinsic to the production of the energetic electrons responsible for the radio and hard X-ray emission, or they can be imposed on the electrons after

acceleration has taken place. Examples of the former mechanism include the current loop coalescence model of Tajima et al. (1987) and the dynamic reconnection discussed by Kliem et al. (2000). However, in general such quasi-periodic reconnection processes that can modulate acceleration, as well as processes in cyclic self-organizing systems, are expected to produce more complex time profiles than found here, as discussed by Aschwanden (1987). He also summarized the properties of different mechanisms for creating modulations such as those seen here, and on those criteria it seems most likely that MHD oscillations explain these observations. Such MHD oscillations of the magnetic loops containing the electrons form a natural source of modulation, that in turn can modulate the electrons themselves, or 
modulate the radiation they produce. An obvious way to modulate the radio emission is to change the magnetic field strength, since it directly affects the gyrosynchrotron emissivity without necessarily modifying the electron velocity distribution. However, the radio and hard X-ray emission are produced by very different emission mechanisms (gyrosynchrotron and bremsstrahlung, respectively) and modulating the magnetic field strength in the radio source does not necessarily change the hard X-ray bremsstrahlung at the other end of loop $\mathrm{L}_{A}$. If the magnetic field in the entire loop is modulated, then it may affect electron precipitation at the western end of loop $\mathrm{L}_{A}$ and thus change the hard X-rays as well.

\subsection{Modulation of gyrosynchrotron radio emission}

The emissivity of the gyrosynchrotron mechanism in the optically thin limit that applies here (frequencies above the peak in the radio spectrum) depends on four parameters: the magnetic field strength $B$; the angle between the magnetic field and the line of sight $\theta$; the nonthermal electron number density $N_{e}$; and the electron energy distribution power-law index $\delta$ (Dulk 1985). We can rule out changes in $\delta$ as the cause of the radio modulation since they would be prominent in the hard X-ray spectra, which show no significant modulation of $\delta$. Changes in $N_{e}$ can be produced by modulation intrinsic to the acceleration mechanism: they will change the radio emissivity linearly and not change the degree of polarization (as long as they do not increase the gyrosynchrotron opacity so as to make the $x$ mode optically thick, in which case the degree of polarization will decrease as $N_{e}$ increases).

Changes in $B$ and $\theta$ can result from MHD effects in the magnetic loops carrying the energetic electrons. Any mechanism that causes the magnetic field strength to change can modulate the radio emission because the gyrosynchrotron emissivity depends strongly on $B$ : emissivity $\propto B^{0.90 \delta-0.22}$ for an $E^{-\delta}$ nonthermal energy distribution (Dulk \& Marsh 1982), so the dependence is $B^{4.3}$ for a typical value $\delta=5$ derived from the HXT data above. Thus a small modulation of $B$ can affect the radio flux strongly. A change in $B$ should also change the degree of polarization of the radio emission, but its dependence on $B$ is much weaker (roughly $B^{0.5}$; Dulk 1985) and consequently the expected $6 \%$ relative change in the degree of polarization corresponding to the $70 \%$ modulation of the total flux might not be measurable; as noted earlier, increasing optical depth can drive the degree of polarization downwards (in the opposite direction to an increase of $B$ ) and complicate the interpretation of any changes.

The gyrosynchrotron emissivity increases with $\theta\left(\propto \sin ^{2.8} \theta\right.$ for $\delta=5$; Dulk 1985), so a $20 \%$ increase in $\sin \theta$ is needed to produce a $70 \%$ modulation of the radio flux. Changes in the viewing angle also affect the degree of polarization but in a more complicated fashion: the $20 \%$ increase in $\sin \theta$ would produce a $20 \%$ decrease in the degree of polarization for a 
harmonic number of order 16 , but a decrease of only about $8 \%$ if the harmonic number is 10 , corresponding to a magnetic field of order $700 \mathrm{G}$. A uniform large-scale change in viewing angle would be required to explain how the radio emission increased everywhere within a resolution element, here of size $10^{\prime \prime}$, or else different changes at different locations within the resolution element will average out.

\subsection{Oscillation modes}

In this section we review the possible modes of oscillation that the environment might impose on electrons travelling along coronal magnetic field lines. Rosenberg (1970) proposed an explanation of QPP by a modulation with variations of the magnetic field in a loop due to its transverse oscillations. This idea has been developed and extended. Possible causes of regular oscillations in coronal loops have been reviewed by Roberts et al. (1984), Aschwanden (1987) and Aschwanden et al. (1999). They include the following:

1. The fast sausage mode, in which a slender flux tube oscillates as a whole via standing symmetric fast-mode waves (Edwin \& Roberts 1983; Roberts et al. 1983). Fast-mode waves can only be freely propagating if the Alfvén speed in the loop is slower than in the surroundings, i.e., the loop must be overdense. Since the loop (in the assumption of cylindrical symmetry) expands and contracts radially, corresponding to a standing wave around the circumference of the tube, the period of the sausage mode is the time taken by a fast mode wave to propagate around the circumference and depends on the radius of the tube:

$$
\tau_{\text {sausage }} \approx 330 \frac{r_{8} \sqrt{n_{10}}}{B} \text { seconds }
$$

where $r_{8}$ is the tube radius in $10^{8} \mathrm{~cm}, n_{10}$ the tube density $\left(10^{10} \mathrm{~cm}^{-3}\right)$ and $B$ the magnetic field in Gauss. We assume that the coronal plasma is fully ionized and $10 \%$ helium (by number). The sausage mode modulates the cross-section area of the loop and thus modulates both $B$ and $n$, by flux conservation. Typically a sausage mode can only exist in the loop if a large number of nodes $(z 10)$ fits along the loop (Edwin \& Roberts 1983), so there will be oscillating regions both near the footpoints and at the looptop.

2. The fast kink mode is the asymmetric counterpart of the sausage mode: the tube is displaced laterally in a sinuous fashion and the period depends on the number of nodes $j-1$ along the length of the loop $\mathrm{L}_{8}\left(10^{8} \mathrm{~cm}\right)$,

$$
\tau_{\text {kink }} \approx 100 \frac{L_{8} \sqrt{n_{10}}}{j B} \text { seconds }
$$


The longest period occurs for the fundamental mode, $j=1$, which is the easiest mode to excite in which the loop apex is disturbed (Roberts et al. 1984). In a given loop kink mode periods are generally much longer than sausage mode periods. Since the kink mode produces a lateral translation of the loop but does not change cross-section area and hence does not modify magnetic field strength or particle density, it is not clear how the kink mode can modulate radio emission. We assume $j=1$ for the calculation of periods.

3. The slow MHD wave versions corresponding to the fast mode oscillations above both have the same period:

$$
\tau_{\text {slow }} \approx 13 \frac{L_{8}}{\sqrt{T_{6}}} \text { seconds }
$$

where $T_{6}$ is the plasma temperature in $10^{6} \mathrm{~K}$.

4. Propagating MHD waves launched at one end of a loop will exhibit a periodic phase (Roberts et al. 1984) with a period in a low- $\beta$ plasma that is 0.6 times less than the fast sausage mode,

$$
\tau_{\text {fast }} \approx 200 \frac{r_{8} \sqrt{n_{10}}}{B} \text { seconds }
$$

This is the non-steady counterpart of the sausage mode: the latter requires a standing-wave pattern of order 10 nodes along the loop, which takes some time to establish, while the propagating version is impulsively generated and short-lived.

5. Torsional waves could be launched by reconnection in a twisted flux tube and form standing waves between the ends of the loop (Tapping 1983). The period for a wave with $m$ windings along the loop is

$$
\tau_{\text {torsion }} \approx 100 \frac{L_{8} \sqrt{n_{10}}}{m B} \text { seconds }
$$

The torsional wave is non-compressive and hence cannot change the value of $B$, but can change the angle $\theta$ between the magnetic field and the line of sight. $m$ is poorly constrained: Tapping (1983) assumed $m \approx 100$ to explain decimetric pulsations with a period of order $1 \mathrm{~s}$, but values as low as $m=1$ are presumably possible. This mechanism produces the largest degree of modulation when the loop is viewed end on $(\theta \approx 0)$ so that changes in $\theta$ are relatively large.

In order to investigate whether these modes produce the periods seen in the NoRH data 
we need to estimate $r, L, n_{e}$ and $T$. We do this separately for sources 2 , 4 (here meaning the western end of loop $\mathrm{L}_{A}$ rather than the location shown in Fig. 3a) and $\mathrm{L}_{B}$. For sources 2 and 4 the relevant loop length is the length of $\mathrm{L}_{A}$. The coordinates of the microwave-emitting ends of the long loop are W72 N23 and W76 N21.5, so the distance between them is $\mathrm{d}=$ $4.5^{\circ}=50 \mathrm{Mm}$. The length of a helical loop with a height $\mathrm{h}=15 \mathrm{Mm}$ is $\pi \sqrt{(d / 2)^{2}+h^{2}}$, so the length is $=92 \mathrm{Mm}$ (for a semicircular loop it would be $\pi / 2 \mathrm{~d}=79 \mathrm{Mm}$ ). This number is consistent with the projected appearance of the loop: Fig. 2c shows that the eastern leg of the loop is $80^{\prime \prime}$ or $\sim 5 \times 10^{9} \mathrm{~cm}$, while the length of the western leg is difficult to estimate due to projection effects (we seem to be looking nearly along the leg). We adopt $92 \times 10^{8}$ $\mathrm{cm}$ for the total length of $\mathrm{L}_{A}$. The loop radius we take from Fig. $2 \mathrm{c}$ to be $6^{\prime \prime}$ or $4 \times 10^{8} \mathrm{~cm}$.

We estimate the densities from the SXT data. We produced temperature and emission measure maps from the multi-filter SXT images following standard procedures. The emission measures are converted to densities by assuming a depth equal to the size of the brightest SXT kernel in loop $\mathrm{L}_{B}, 8 \times 10^{8} \mathrm{~cm}$. We find that the density in the long loop $\mathrm{L}_{A}$ increases from $0.9 \times 10^{10} \mathrm{~cm}^{-3}$ during the rise of the first burst to $1.1 \times 10^{10} \mathrm{~cm}^{-3}$ during the decay of the first burst, and $2.3 \times 10^{10} \mathrm{~cm}^{-3}$ during the second burst. In the short compact loop $\mathrm{L}_{B}$, the average density during the first burst is $2.4 \times 10^{10} \mathrm{~cm}^{-3}$, with the peak density being $15 \times 10^{10} \mathrm{~cm}^{-3}$; during the second burst the average density in $\mathrm{L}_{B}$ is $4 \times 10^{10} \mathrm{~cm}^{-3}$ with the peak being $20 \times 10^{10} \mathrm{~cm}^{-3}$. Temperatures are in the range 10 to 16 megakelvin (MK); we use $10 \mathrm{MK}$ for the estimation of the slow-mode oscillation periods.

To estimate the magnetic field we fitted the observed radio spectrum using the Ramaty code (Ramaty 1969; Ramaty et al. 1994) and find a magnetic field of 720 G that we assume applies to source 2, which is the dominant radio source. Since we only have measurements of the radio flux at the other end of the loop at two frequencies, we cannot use the same approach to determine $B$ in source 4 . We can assume that the magnetic field is significantly weaker at the other end of loop $\mathrm{L}_{A}$, since the hard X-ray images indicate that precipitation of energetic electrons overwhelmingly occurs at source 4, and the radio images during the period of oscillations indicate that radio emission from source 4 is 20 times weaker than at source 2. Both these considerations suggest that $B$ is considerably weaker in source 4 during the oscillations. We will adopt a value for $B$ in source 4 that is half the field strength in source 2, i.e., $360 \mathrm{G}$, but there is at least $30 \%$ uncertainty in this value. Our assumed value is consistent with that of Asai et al. (2001) who estimate a field strength of $300 \mathrm{G}$ at the top of the compact loop seen by SXT in this location (source 4) using a potential-field extrapolation of a magnetogram obtained on November 6 (longitudinal magnetograms for regions at the limb are not adequate for estimating magnetic field strengths). Note that the turnover frequency during the rise phase of the first burst may be higher in source 4 than in source 2, which would imply that the magnetic field is stronger in source 4 at that stage of 
the event. The abrupt drop in radio emission from source 4 in the first burst may indicate reconnection of the fieldlines of loop A to different footpoints in the region of source 4 with a weaker magnetic field. However, since no oscillations are seen in source 4 during the first burst, only the apparently weaker field during the second burst is relevant for calculating the oscillation periods.

In Table 1 we use these parameters to investigate the oscillation periods during the rise of the first burst, the decay of the first burst, and the second burst at three locations: the eastern end of the long loop $\mathrm{L}_{A}$ (source 2), the western end of the long loop $\mathrm{L}_{A}$ (source 4), and the bright soft $\mathrm{X}$-ray loop $\mathrm{L}_{B}$. Taking into account the estimates of the magnetic field strength at the top of loop $\mathrm{L}_{B}$ by Asai et al. $(2001, \approx 300 \mathrm{G})$, we assume that the magnetic field in $\mathrm{L}_{B}$ is the same as in the adjacent western end of $\mathrm{L}_{A}(360 \mathrm{G})$, bearing in mind the uncertainty in this value. The density is assumed to be the same at both ends of $\mathrm{L}_{A}$.

Periods in $\mathrm{L}_{B}$ are generally longer than in $\mathrm{L}_{A}$, even though the dimensions of $\mathrm{L}_{B}$ are smaller, because the higher density in $\mathrm{L}_{B}$ means slower MHD wave speeds. For the same reason, the oscillation periods increase with time as density in the corona increases. If the time structure in the first burst is related to the modulation then the fact that it has shorter timescales than the oscillations in the second burst would be consistent with a lower density in the loop earlier in the flare. However, for the parameters used the periods shown for source 2 are all either too short or too long, while the period for the sausage mode in source 4 is of the right order to explain the observed modulation, as is the period of the torsional wave in $\mathrm{L}_{A}$ for $m \approx 2-3$.

In Table 1 we show different periods for the sausage mode at the two ends of $\mathrm{L}_{A}$ because of the inference that the magnetic field is different at the ends of the loop: $\tau_{\text {sausage }}$ depends on the loop radius which, under the assumption of magnetic flux conservation, will be different at the two ends of the loop. While the period of the sausage mode does not depend on the loop length, the properties of the mode are derived under the assumption of cylindrical symmetry (Edwin \& Roberts 1983), i.e., the period of the sausage mode is the same everywhere along the loop, permitting a standing wave pattern with multiple nodes along the loop length. This pattern cannot be sustained when the radius of the loop and hence the period of the mode varies along the loop. Further, the standing mode pattern cannot be established in a timescale shorter than the time taken by a fast-mode wave to propagate along the loop and back again, which is of order $20 \mathrm{~s}$ for $\mathrm{L}_{A}$. Since bursts 1 and 2 are both about this long, it does not seem likely that there is sufficient time to set up a standing sausage-mode pattern. Thus the impulsively-generated propagating fast mode wave is a more likely explanation for the modulation than the standing-wave sausage mode. By contrast, the kink mode period depends on the length of the loop and thus should be the same everywhere along the loop, 
but the kink mode periods are too long to explain the oscillations. If the natural periods at the two ends of loop $\mathrm{L}_{A}$ are different, the fact that the observed period during the second burst is the same in radio at the eastern end of $\mathrm{L}_{A}$ and in X-rays at the western end of $\mathrm{L}_{A}$ implies that the modulation is not taking place independently at the two locations, since they would then probably have different periods, but rather is produced at one location and then propagates to the other: since the wave propagation times are all much longer than the observed delays, it must be accelerated electrons modulated in the oscillating region that propagate to the sites where they radiate radio and hard X-ray emission, respectively.

\subsection{Overall scenario}

This event fits into the class of "interacting loop" (also called "double loop") events identified by Hanaoka (1996, 1997, 1999b,a); see also Kundu \& Shevgaonkar (1985) and Kundu et al. (2001). This class of flares is characterized by radio images showing two sources separated by a large distance (the original identifying feature), hard X-ray and radio light curves with many subsidiary peaks, often showing quasi-periodic features and delays at the remote source, and X-ray emission located at only one of the radio sources. This event shows all of these features. It differs from most Hanaoka events in that the radio emission located at the dominant X-ray source is not the dominant radio source: in this event the remote radio source is stronger.

The interpretation of these phenomena is that the flares involve an interaction between two magnetic loop systems: a compact loop which is the main energy release site and dominates the X-ray emission, and a longer loop connecting the main energy release site to the remote site. In some events more than two sets of field lines seem to be involved (cf. Grechnev \& Nakajima 2002). Hanaoka (1996, 1997, 1999b) speculated that the interaction is driven by the emergence of new magnetic flux into a region of existing loops. In this event, the two interacting loops are $\mathrm{L}_{A}$ and $\mathrm{L}_{B}$. In the initial burst, radio emission from the $\mathrm{L}_{B}$ region cuts off earlier than that from source 2 ; we speculate that this is because of disconnection of the field lines from the source of energetic electrons. The same phenomenon was inferred in another Hanaoka event by Kundu et al. (2001). This disconnection may be due to reconnection: Fig. 3 shows that the radio emission from source 4 comes from the loop $\mathrm{L}_{B}$ during the first burst, but from a location just to the west of $\mathrm{L}_{B}$ during the second burst, which is consistent with the idea that field lines initially connected $\mathrm{L}_{B}$ to source 2 but were disconnected during the first burst.

The quasi-periodic modulation can be intrinsic to the source of the energetic electrons, or it can be imposed on the electrons by a property of the loop. Asai et al. (2001) proposed 
that the modulation is due to oscillations of coronal loops. They also proposed that "the origin of QPP in microwaves and hard X-rays is the modulation of the acceleration/injection of nonthermal electrons". However, the modulation of the radio emission of up to $70 \%$ is much stronger than the modulation of the hard X-rays (Fig. 7). This observation suggests that the modulation is a property intrinsic to the radio emission; for example, a highlymodulated nonthermal $>300 \mathrm{keV}$ electron population may dominate the radio emission and contribute weakly to the $<50 \mathrm{keV}$ hard X-rays, which would be dominated by another $<$ $300 \mathrm{keV}$ population that is unmodulated or only weakly modulated.

Depending upon the injection process, trapping of microwave-emitting electrons within a magnetic loop can produce some difference between hard X-ray and microwave time profiles. During the second burst, the trapping time seems not to exceed 1-2 seconds, otherwise we would not see the similarity in short-duration features in these two emissions. The trapping effect will generally lead to a decrease in the modulation depth.

The locations of the modulated radio and X-ray sources at opposite ends of a long loop imply that the electrons responsible for both forms of emission are travelling along the loop $\mathrm{L}_{A}$. The vicinity of source 4 is the most likely place for electron acceleration to take place, because the X-ray data indicate that this is the brightest X-ray source and hence most of the flare energy is released there; it is also the site where two separate loops involved in the flare $\left(\mathrm{L}_{A}\right.$ and $\left.\mathrm{L}_{B}\right)$ may be interacting. However, it is then hard to understand why the radio modulation at source 2 , some $10^{10} \mathrm{~cm}$ away, is much stronger than the X-ray modulation at the much closer source 4. Further, the X-rays show no modulation during the first peak when the radio emission from source 2 does show fine structure: since the X-ray source could not be the source of the modulation in the first burst, it seems unlikely to be the source of modulation in the second burst.

The fact that fine structure is seen first in source 2 during the first burst, and the deepest modulation is seen in source 2 during the second burst, suggests that the pulsations are associated with that location. However, the delay of the radio emission in source 2 relative to both the hard X-rays and radio emission in source 4 during the second burst suggests, on the grounds of causality, that the modulation must arise in source 4 . In the latter case it is difficult to understand how the radio modulation at source 2 can be so pronounced in the second burst, since the electrons must travel $10^{10} \mathrm{~cm}$ to reach source 2 and a modest velocity or pitch angle dispersion will result in a range of propagation times that could smooth out modulations in the electron density induced at source 4. An electron with zero pitch angle and an energy of $200 \mathrm{keV}$ takes $0.48 \mathrm{~s}$ to travel $10^{10} \mathrm{~cm}$, while a $1 \mathrm{MeV}$ electron takes $0.35 \mathrm{~s}$. For an electron with $30^{\circ}$ pitch angle the times are longer by about $15 \%$; at $45^{\circ}$ pitch angle times are longer by $41 \%$. If indeed the origin of the modulations is located 
at source 4 and the electrons propagate to source 2 where they produce the modulated radio emission, then the inferred delay of order $0.5 \mathrm{~s}$ implies that the radio emission is dominated by relatively low-energy electrons $(\sim 200 \mathrm{keV})$. At the $1 \mathrm{~s}$ time resolution of the data for the second burst it may be difficult to see the subsecond smoothing effects of energy and pitch angle spreads provided that they are not extreme. However, it remains difficult to understand how the depth of modulation can be greater in the radio emission from source 2 than it is in the X-ray or radio emission from source 4, closer to the energy release site, since any spread in energy or pitch angle would be expected to have the effect of diminishing the modulation depth at the remote site. Further, electron trapping would be expected to build up a population of electrons in the loop over time that would lose its memory of the modulation and still contribute to radio emission from the footpoints thanks to pitch angle scattering repopulating the loss cone. Since the number of trapped electrons should increase with time, they should have the effect of decreasing the relative modulation of later peaks in the wave train, yet the fourth peak in burst 2 is far more pronounced in the radio emission from source 2 than in radio or hard X-rays from source 4, and further it appears that source 2 leads source 4 for this peak. Thus the scenario in which the modulation is imposed on the accelerated electrons in the energy release site near source 4 does not straightforwardly explain all the properties of the modulations in the remote source.

On the other hand, a wave train propagating along the loop can modulate the emission at the two ends of the loop provided that it can maintain phase coherence over the length of the loop. Since none of the modes discussed in the preceding subsection are fast enough to get from one end of the loop to the other in the observed delay time of $0.5 \mathrm{~s}$ (Table 1), this explanation requires that the disturbance is launched almost exactly in the middle of the loop and propagates towards both footpoints, maintaining phase coherence on both sides of the loop.

\section{CONCLUSIONS}

Using multi-spectral imaging and non-imaging data, i.e., microwave data of Nobeyama Radioheliograph and Polarimeters, soft and hard X-ray Yohkoh data, SOHO/EIT and SOHO/MDI images as well as total flux soft X-ray GOES data, we have made a detailed study of the impulsive flare event that occurred on November 10, 1998. The flare is consistent with the class of interacting-loop flares. Important observational constraints on physical parameters are obtained from a study of the oscillations. The coordinated study of all available data allows us to reveal important processes which governed the flare events, and to carry out diagnostics of plasma parameters in the flare regions. 
The most striking phenomenon was the second burst with unusually high microwave pulsations. Asai et al. (2001) discussed these pulsations in terms of two sources at opposite ends of a long loop, showing that the radio emission at one end and the hard X-ray emission at the other end were both modulated in the same way. In this paper we show in addition that (i) at least five distinct radio sources with very different time profiles can be identified, including emission from the long loop connecting the modulated radio and X-ray sources; (ii) radio fine structure also appears to be present in source 2 during the first burst of the flare, but with timescales shorter than the modulation period in the second burst; (iii) radio modulations are seen in source 4 during the second burst, but at a level some 20 times weaker than in source 2; (iv) these radio modulations in source 4, like the hard X-ray modulations at the same location, appear to lead the modulations in source 2 by about $0.5 \mathrm{~s}$, but all have the same period.

We investigate possible origins of the modulation period. The regularity of the modulation suggests an oscillation of magnetic loops rather than an origin intrinsic to the acceleration mechanism, although the latter cannot be ruled out. For the physical conditions believed to be present in the sources, either propagating fast mode MHD waves, which change the magnetic field strength in the loop, or torsional waves, which change the angle between the magnetic field and the line of sight, could produce oscillations with timescales close to those observed. The increase in fine structure timescale from the first to the second burst is expected in both models due to the increase in density in the loop $\mathrm{L}_{A}$ due to chromospheric evaporation, which leads to a decrease in the Alfvén speed. Impulsive reconnection is an event that could plausibly generate a burst of propagating fast mode waves. In common with other events of this class, the radio light curves of spatially separated sources show effects that are consistent with the disconnection of the magnetic link between them at a time close to the peak of the impulsive phase that could be explained by a large-scale rearrangement of magnetic field lines due to reconnection. If this is the correct explanation, then this flare seems to show a sequence of several episodes of impulsive reconnection.

However, we are unable to find a self-consistent picture that explains why the depth of modulation is much larger in source 2 despite being delayed relative to the modulation in source 4 . If the modulations are produced by a wave train launched somewhere near the loop top, it seems implausible that this wave train could propagate down both sides of the loop maintaining phase coherence to within the observed delay time of $0.5 \mathrm{~s}$ over propagation times of order $10 \mathrm{~s}$ for fast mode waves. This suggests instead that the oscillations modulate the source of accelerated electrons. If the energy release and the modulation originate in source 4 and affect the accelerated electron population which then propagates $10^{10} \mathrm{~cm}$ to source 2, then any spread in energy or pitch angle, or the accumulation of electrons trapped in the loop, would be expected to diminish the degree of modulation shown by the electrons 
when they arrive at source 2 , yet the observed modulation is stronger at source 2 than at source 4 .

M. R. Kundu and V. V. Grechnev wish to thank the staff of NAOJ and NRO (Japan) for their help and hospitality during their stays at the observatories, and for recovering the high-cadence raw data. The authors are grateful to V. Garaimov and A. Altyntsev for fruitful discussions. This research was supported by NSF grants ATM 99-90809 and INT-98-19917, NASA grant NAG 5-8192, Russian RFBR grants 00-15-96710, 00-02-16819 and 01-02-16290, and INTAS grant 2000-00543. We gratefully acknowledge the open-data policies of NoRH, the ISAS/NASA satellite Yohkoh and the ESA/NASA satellite SOHO and their instrument teams which make multi-wavelength research such as this much easier. 
Table 1. Oscillation periods

\begin{tabular}{|c|c|c|c|c|c|c|c|c|c|c|}
\hline Event & $\begin{array}{l}B \\
G\end{array}$ & $\begin{array}{c}n_{e} \\
10^{10} \mathrm{~cm}^{-3}\end{array}$ & $\begin{array}{l}\text { Length } \\
10^{8} \mathrm{~cm}\end{array}$ & $\begin{array}{l}\text { Radius } \\
10^{8} \mathrm{~cm}\end{array}$ & $\begin{array}{c}\mathrm{v}_{A} \\
\mathrm{~km} \mathrm{~s}^{-1}\end{array}$ & $\begin{array}{c}\tau_{\text {saus }} \\
\mathrm{s}\end{array}$ & $\begin{array}{c}\tau_{k i n k} \\
\mathrm{~s}\end{array}$ & $\begin{array}{c}\tau_{\text {slow }} \\
\mathrm{s}\end{array}$ & $\begin{array}{c}\tau_{\text {fast }} \\
\mathrm{s}\end{array}$ & $\begin{array}{c}\tau_{\text {tor }} \\
\mathrm{s}\end{array}$ \\
\hline \multicolumn{11}{|l|}{ Source 2} \\
\hline 1st burst, rise & 720 & 0.9 & 92 & 4 & 15400 & 1.7 & 12 & 380 & 1.1 & $12 / m$ \\
\hline 1st burst, fall & 720 & 1.3 & 92 & 4 & 12800 & 2.1 & 15 & 380 & 1.3 & $15 / m$ \\
\hline 2nd burst & 720 & 2.4 & 92 & 4 & 9400 & 2.8 & 20 & 380 & 1.7 & $20 / m$ \\
\hline \multicolumn{11}{|l|}{ Source 4} \\
\hline 1st burst, rise & 360 & 0.9 & 92 & 4 & 7700 & 3.4 & 24 & 380 & 2.2 & $24 / m$ \\
\hline 1st burst, fall & 360 & 1.3 & 92 & 4 & 6400 & 4.2 & 30 & 380 & 2.6 & $30 / m$ \\
\hline 2nd burst & 360 & 2.4 & 92 & 4 & 4700 & 5.6 & 40 & 380 & 3.4 & $40 / m$ \\
\hline \multicolumn{11}{|l|}{ Loop $\mathrm{L}_{B}$} \\
\hline 1st burst & 360 & 15.0 & 15 & 3 & 1900 & 11 & 16 & 62 & 6.4 & $16 / m$ \\
\hline 2nd burst & 360 & 20.0 & 15 & 3 & 1600 & 12 & 19 & 62 & 7.5 & $19 / m$ \\
\hline
\end{tabular}




\section{REFERENCES}

Altyntsev, A., Grechnev, V., \& Hanaoka, Y. 1998, Solar Phys., 178, 137

Altyntsev, A. T., Grechnev, V. V., Nakajima, H., Fujiki, K., Nishio, M., \& Prosovetsky, D. V. 1999, Astron. Astrophys. Supp. Ser., 135, 415

Asai, A., Shimojo, M., Isobe, H., Morimoto, T., Yokoyama, T., Shibasaki, K., \& Nakajima, H. 2001, Astrophys. J. (Lett.), 562, L103

Aschwanden, M. J. 1987, Solar Phys., 111, 113

Aschwanden, M. J., Fletcher, L., Schrijver, C. J., \& Alexander, D. 1999, Astrophys. J., 520, 880

Dulk, G. A. 1985, Ann. Rev. Astron. Astrophys., 23, 169

Dulk, G. A. \& Marsh, K. A. 1982, Astrophys. J., 259, 350

Edwin, P. M. \& Roberts, B. 1983, Solar Phys., 88, 179

Grechnev, V. V. \& Nakajima, H. 2002, Astrophys. J., 566, 539

Hanaoka, Y. 1996, Solar Phys., 165, 275

—. 1997, Solar Phys., 173, 319

—. 1999a, Publ. Astron. Soc. Japan, 51, 483

Hanaoka, Y. 1999b, in Nobeyama Symposium on Solar Physics with Radio Observations, ed. T. S. Bastian, N. Gopalswamy, \& K. Shibasaki (Nobeyama Radio Observatory), 229

Kliem, B., Karlický, M., \& Benz, A. O. 2000, Astron. Astrophys., 360, 715

Kundu, M. R., Grechnev, V. V., Garaimov, V. I., \& White, S. M. 2001, Astrophys. J., 563, 389

Kundu, M. R. \& Shevgaonkar, R. K. 1985, ApJ, 297, 644

Nakajima, H., Kosugi, T., Kai, K., \& Enome, S. 1983, Nature, 305, 292

Ramaty, R. 1969, Astrophys. J., 158, 753

Ramaty, R., Schwartz, R. A., Enome, S., \& Nakajima, H. 1994, Astrophys. J., 436, 941 
Roberts, B., Edwin, P. M., \& Benz, A. O. 1983, Nature, 305, 688

—. 1984, Astrophys. J., 279, 857

Rosenberg, H. 1970, Astron. Astrophys., 9, 159

Tajima, T., Sakai, J., Nakajima, H., Kosugi, T., Brunel, F., \& Kundu, M. R. 1987, Astrophys. J., 321, 1031

Tapping, K. F. 1983, Solar Phys., 87, 177

White, S. M., Thejappa, G., \& Kundu, M. R. 1992, Solar Phys., 138, 163 


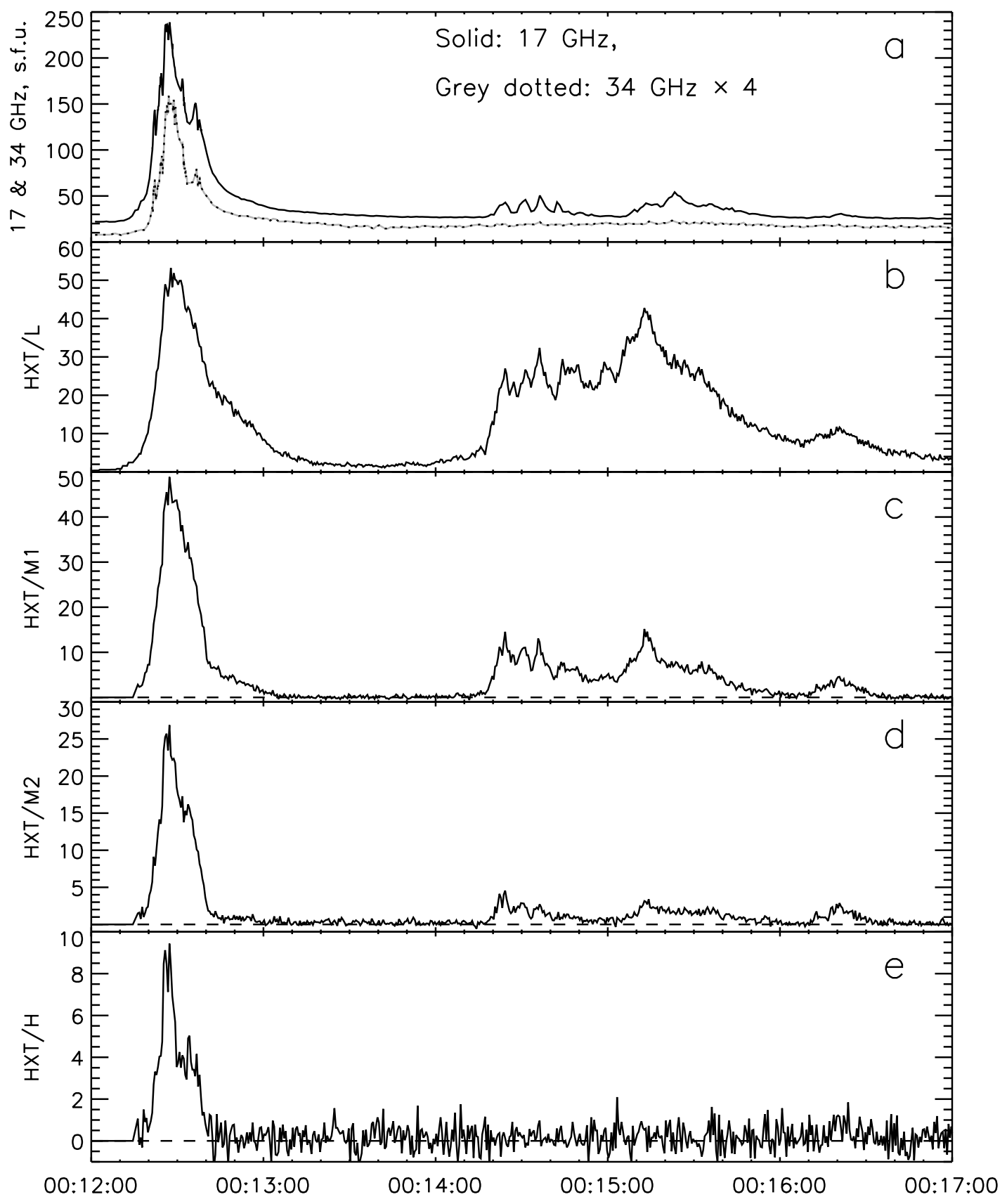

Fig. 1.- Microwave (a, NoRH) and hard X-ray (b-e, Yohkoh/HXT) total flux time profiles of the flare of November 10, 1998. The black solid curve in panel (a) shows the total intensity at $17 \mathrm{GHz}$, while the gray dotted curve shows the total intensity at $34 \mathrm{GHz}$ multiplied by 4 . 


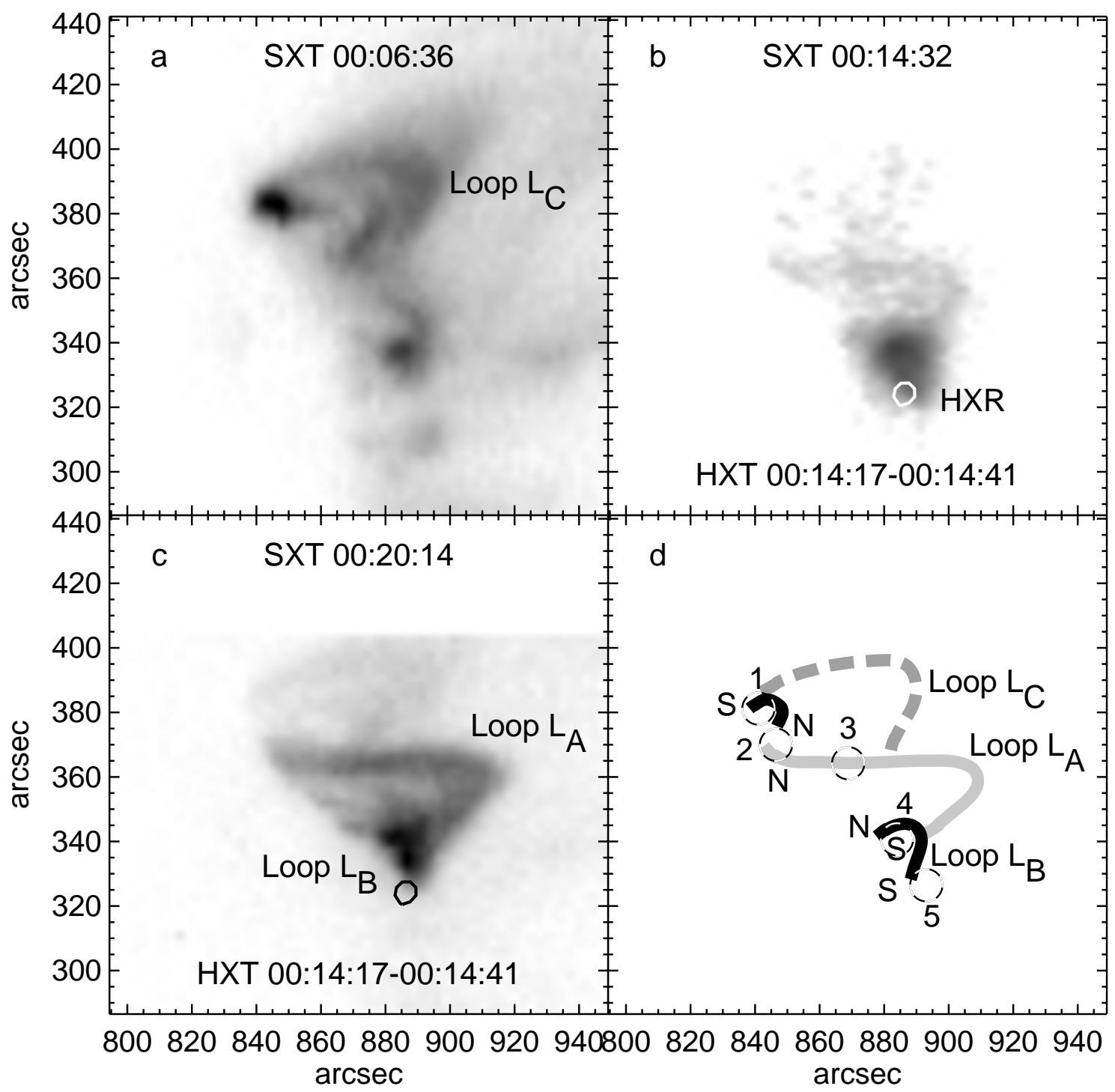

Fig. 2.- Yohkoh/SXT images of the coronal structure observed in soft X-rays (all negative): a) before the event, at 00:06:18-00:10:28 UT, b) one frame obtained at 00:14:32 during the oscillatory second burst, c) after the event, at 00:20:14. A contour in panels (b) and (c) shows the $50 \%$ level of a hard X-ray image obtained during the second burst (M2 band, 00:14:17-00:14:41). Axes show arc seconds from the solar disk center. The main soft X-ray loops are labeled $\mathrm{L}_{A}$ (long faint loop not visible prior to the flare), $\mathrm{L}_{B}$ (the compact loop that is the brightest soft X-ray source), and $\mathrm{L}_{C}$ (long preflare loop in the north). (d) A sketch of the coronal configuration. Major loops visible in soft X-rays are shown: the three loops labelled in other panels together with the compact loop in the north-east that is bright in radio and soft X-rays prior to the flare. The magnetic polarity for each loop footpoint is indicated ("N" for north, "S" for south). The central parts of the microwave-emitting regions $1-5$ are outlined by dashed contours. 


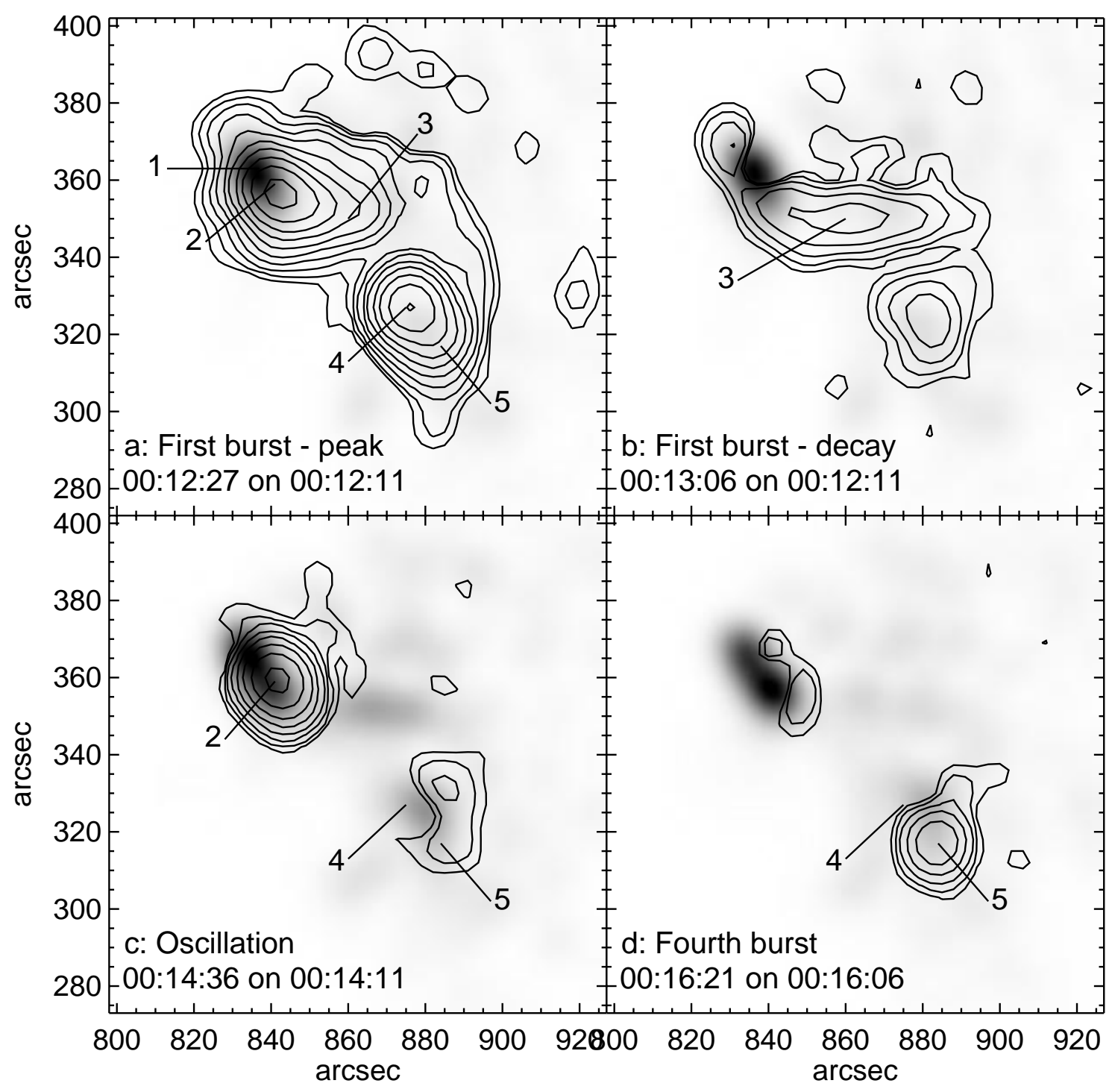

Fig. 3.- $17 \mathrm{GHz}$ NoRH radio images of the flare at selected times. In each case the contours represent an image that is the difference between the later and the earlier time shown in each panel. The earlier image that is subtracted is shown by the underlaying greyscale image. Distinct radio sources are labelled by numbers. The images are 1-second snapshots with a restoring beam of $10^{\prime \prime}$. Contour levels in each image are at $4 \times 10^{4} \mathrm{~K}$ times powers of 2 . 


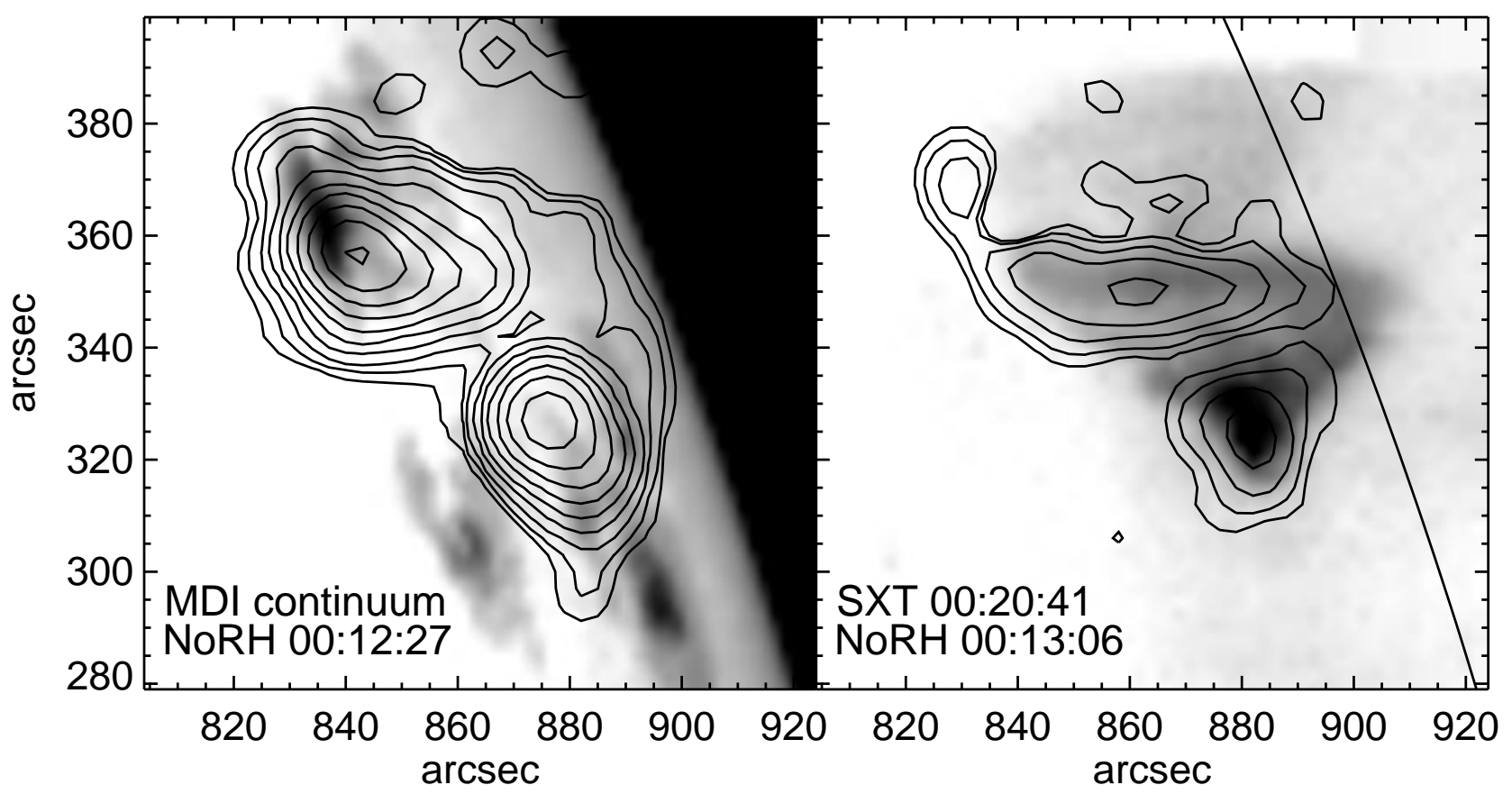

Fig. 4.- Comparison of the location of radio sources (contours) with optical features (left panel, MDI continuum image from 00:12:00) and soft X-ray features (right panel, SXT image from the period 00:19:48 to 00:21:34 with the solar limb shown as a solid line). The radio images are difference images, as described in the caption to Fig. 3, and contour levels are plotted at $25000 \mathrm{~K}$ times powers of 2 . The right panel shows that radio source 3 is coincident with the eastern leg of the soft X-ray loop $\mathrm{L}_{A}$ linking sources 2 and 4, while radio emission from the western leg can be seen in the extension of the contours at source 4 up the leg. 


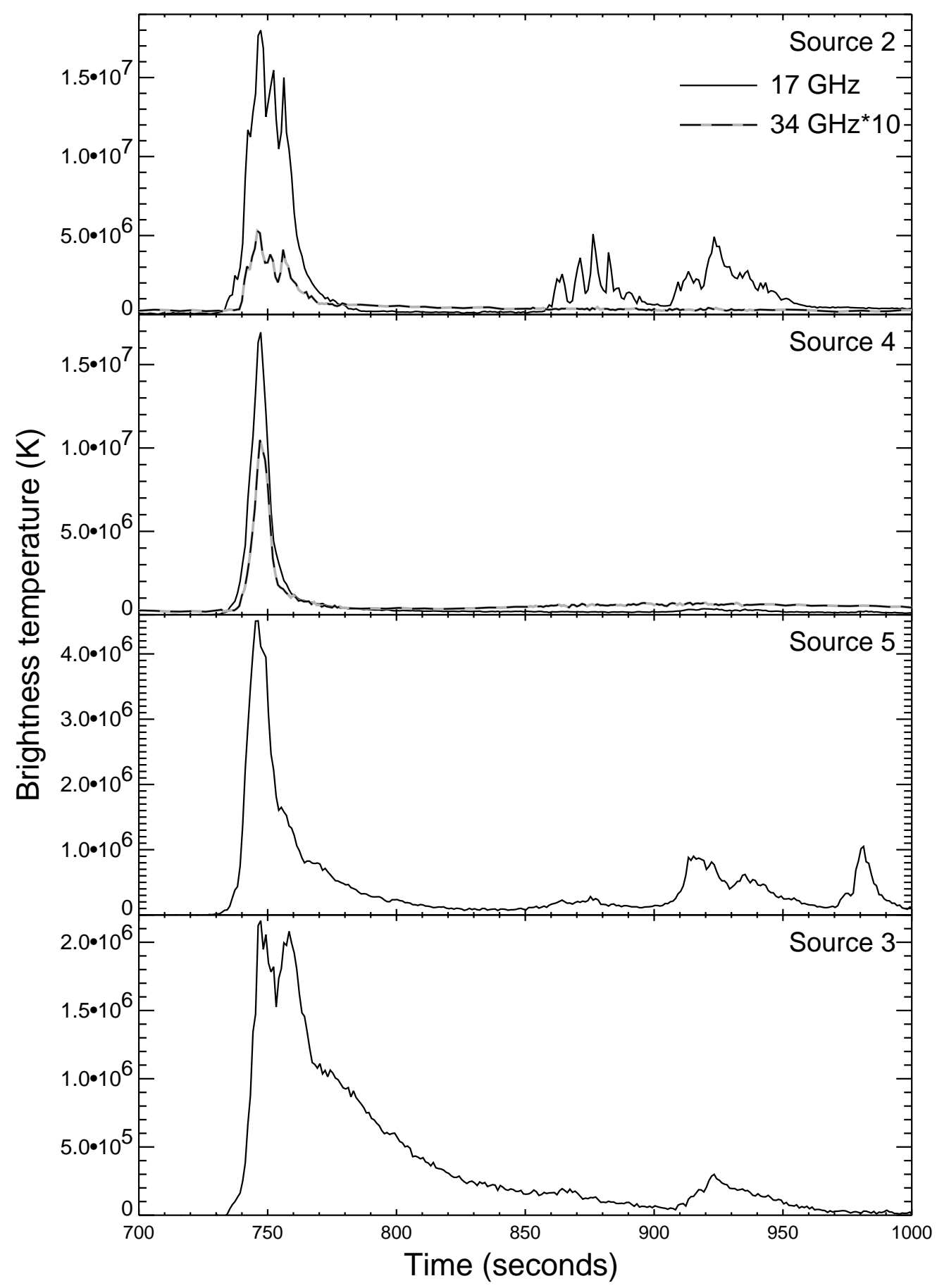

Fig. 5.- Radio light curves for four sources identified in Fig. 3. The quantity plotted is the mean brightness temperature averaged over a square region of side $9^{\prime \prime}$ centered on the brightest pixel in the source region. The solid black line in each panel is the $17 \mathrm{GHz}$ brightness temperature, while in the top two panels the $34 \mathrm{GHz}$ brightness temperature for the sources, multiplied by 10 , is plotted as a grey line with black dashes. 

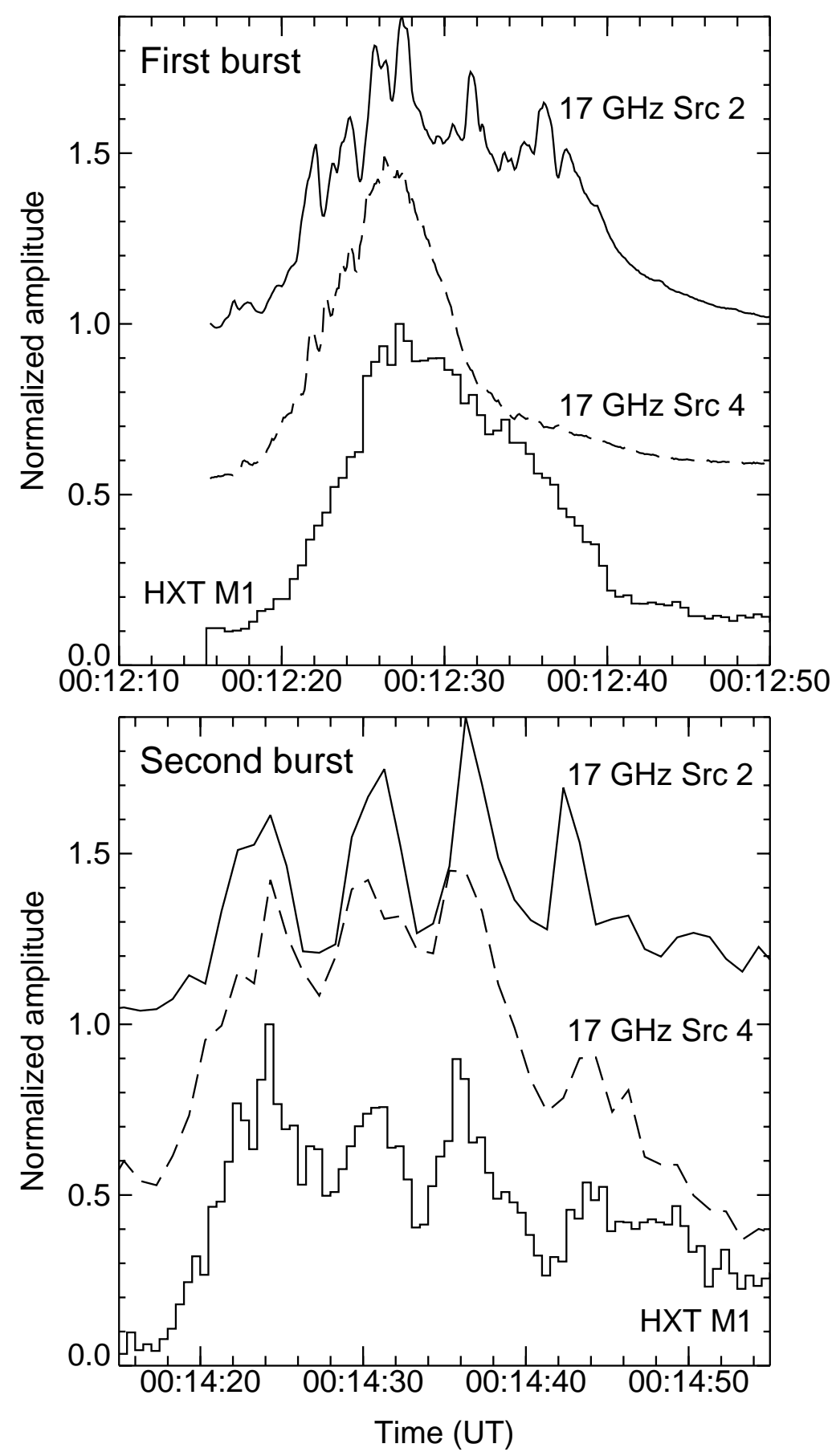

Fig. 6.- Detailed time profiles of the first and second impulsive bursts at $17 \mathrm{GHz}$ and in hard X-rays. The curves are all normalized to a common scale such that they range from 0 to 1 , and adjacent curves are offset from one another for clarity. Both panels show 40 second periods. The curves show the $17 \mathrm{GHz}$ emission from source 2 (solid curve), the 17 GHz emission from source 4 (dashed curve), and the HXT M1 hard X-ray channel (23-33 $\mathrm{keV}$, solid histogram). The time resolution of the hard X-ray data is 0.5 seconds, while the radio fluxes are obtained from uniformly-processed $0.1 \mathrm{~s}$ data for the first burst and 1 second data for the second burst (when no high-time-resolution data are available). 


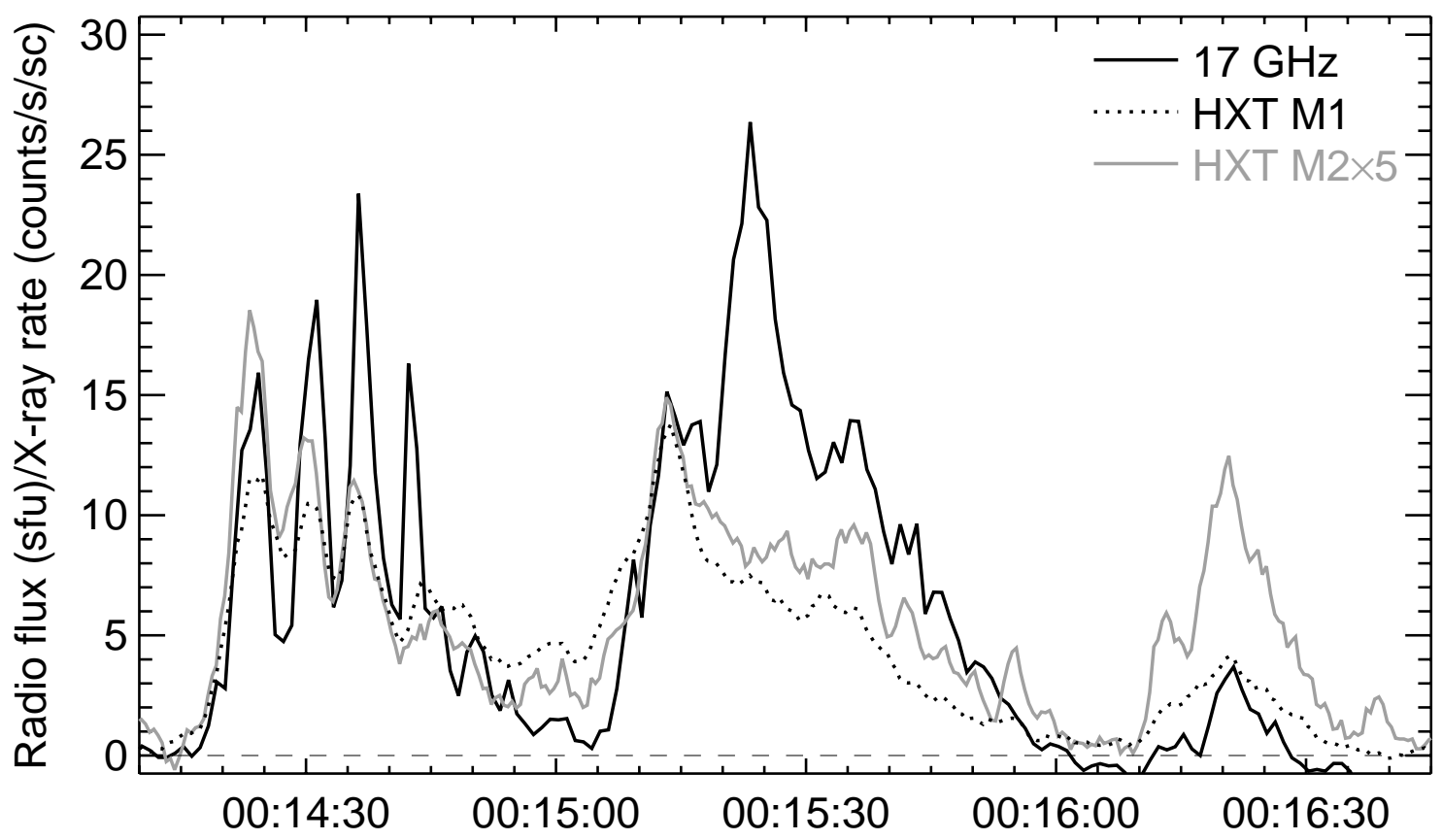

Fig. 7.- Microwave and hard X-ray time profiles of the second, third, and fourth bursts: black solid-17 GHz, dotted-HXT/M1 channel, gray solid-HXT/M2 channel multiplied by 5 . HXT data are smoothed over 5 points. A background level preceding the bursts is subtracted, and the horizontal dashed line shows the zero level. 